\title{
Pubertal period influence on sports results among 13-14 year-old boys swimmers
}

\author{
Olga Yuzhikova ${ }^{1}$, Elena Kashirskaya $^{2}$, Alexander Dorontsev ${ }^{2 *}$, Victoria Gladenkova ${ }^{1}$, Svetlana Pavlova $^{3}$ \\ ${ }^{1}$ Astrakhan State Technical University, 414056, Astrakhan, Russia \\ ${ }^{2}$ Astrakhan State Medical University, 414000, Astrakhan, Russia \\ ${ }^{3}$ Astrakhan State University, 414056, Astrakhan, Russia
}

\begin{abstract}
The aim of this research work was to reveal specific peculiarities of young swimmers training, taking into account their pubescence in terms of the long-term training concept. During the research it was defined that methodological support of spots training for a long-term period should contain not only prognostic model of prospective results achievement. It also should analyze individual peculiarities of pubertal period. During the research we used the official results of competitions protocols, the results of general and special physical readiness tests, resume of the leading swimming coaches. For statistical data handling we used quantitative variables, presented in a form \pm of a standard error of the mean value $(\mathrm{M} \pm \mathrm{m})$. The results were considered as statistically significant in terms of $p<0,05$. Correlation analysis is realized using Pearson correlation coefficient. The held research helped to define typical characteristics of pubertal period in teen-agers swimmers, timely correct the training process. It was stated that athletes adaptation to the training and competitive loads in pubertal period was characterized by different degree of sports results increase and correlated with the intensity of hormonal changes. The boys with distinct features of pubertal period were the leaders at short distances, but showed coordinating abilities decrease. The athletes, whose somatic-gender changes didn't have extreme indices or had prolonged duration, showed stable sports results increase mainly at long distances.
\end{abstract}

\section{Introduction}

Typical specificity of a long-term sports training happens during pubescence cycle [1]. We see heterochronicity of an athlete's functional and somatic systems formation $[2,4]$. As a result of this concept of trainings, their correct content for a long-term period, the future prospective qualities determination should be realized based on individual characteristics of athletes' morpho-functional formation $[3,8,11]$. Special attention in physical load planning in teenagers during morpho-functional changes is caused by endocrine status transformation. It is necessary to pay attention to the coordinated work of cardio-respiratory system [5,6,7,8,9]. According to foreign literature, the authors [10,13] have the opinion that in young athletes training it is necessary to pay great attention to the level of physical load variation. In a modern concept of teen-age sports development many specialists $[12,14,15,16,17]$ consider selection realization reasonable after pubertal period. In this connection there appeared the necessity to study the dynamics of sports indices in pubertal period boys (13-14 years-old), who go in for swimming. For the research work it is interesting to study unstable sports results at different distances during competitive and preparatory periods of a yearly training cycle in athletes of this age group.

\section{Materials and Methods}

The materials for the research were the results of training lessons in swimming among the students of Astrakhan sports school of water kinds of sport named after B.N. Skokov, Russia, who had sports qualification from the $3^{\text {rd }}$ and the $1^{\text {st }}$ category. During the held work we analyzed the dynamics of physical qualities development and sports results, anthropometric results, functional tests indices, start exercises fulfillment and speed turns. Statistical analysis was realized using application program package (Statistica 12.0, the USA). The validity of differences was estimated according to Student-t-criterion. The differences were considered valid in case $p<0,05$. Correlation analysis is realized using Pearson correlation coefficient. The research works were held on the basis of Astrakhan sports school of water kinds of sport named after B.N. Skokov, Astrakhan State Technical University and Astrakhan State Medical University in 2018-2019, Russia. All control normatives in swimming were held at shorts water.

\section{Results and Discussion}

\footnotetext{
${ }^{*}$ Corresponding author: aleksandr.doroncev@ rambler.ru
} 
According to medical check-up 2018/19 it was defined that among 32 13-14 year-old boys in the first group ( $\mathrm{n}_{1}-9$ ) 9 people pubertal period was characterized by high tempo of extremities and body growth $-17 \pm 2,9 \%$, in the second group $\left(\mathrm{n}_{2}-16\right) 16$ athletes during pubertal period had moderate increase of body and extremities length $-10,7 \pm 1,9 \%$, in the third group $\left(n_{3}-7\right) 7$ teen-agers had slow changes of growth indices $-4,2 \pm 0,9 \%$.

Analyzing the dynamics of effectiveness during 50 meters distance overcoming with free style it was defined that among teen-agers of $\left(n_{1}-9\right)$ group there was considerable increase of straight parts overcoming; the first 25 meters $14.57 \pm 0,38 \mathrm{~s}$. till $13.00 \pm 0,11 \mathrm{~s}$., the $2^{\text {nd }} 25$ meters $16,20 \pm 0,24 \mathrm{~s}$. till 15,33 $\pm 0,12 \mathrm{~s}$. at the beginning of the academic year and at the end of the academic year. At 100 meters distance straight parts overcoming at the beginning of the research was the following: the $1^{\text {st }} 25$ meters $14.27 \pm 0,44 \mathrm{~s}$., the $2^{\text {nd }} 25$ meters $16.50 \pm 0,37 \mathrm{~s}$., the third part $17,49 \pm 0,44$ and the final 25 meters $16.00 \pm 0,28 \mathrm{~s}$. According to the final protocols at the end of the academic year the indices were the following: the $1^{\text {st }}$ part $14,01 \pm 0,35 \mathrm{~s} .(\mathrm{p}<0,005)$, the $2^{\text {nd }}$ line $16,00 \pm 0,23 \mathrm{~s}$. $\quad(\mathrm{p}<0,005)$, the $3^{\text {rd }}$ part

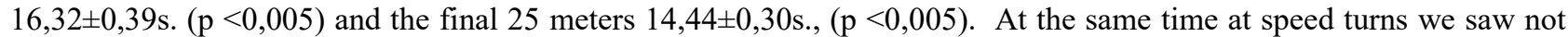
stable roll fulfillment and the intensity of repulsion as a result of spatial characteristics of body position mistakes.

In $\left(\mathrm{n}_{2}-16\right)$ group there was a valid results increase at 100 meters distance, from 1: 12,35 $\pm 0,24 \mathrm{~s}$. to $1: 10,31 \pm 0,19 \mathrm{~s}$.

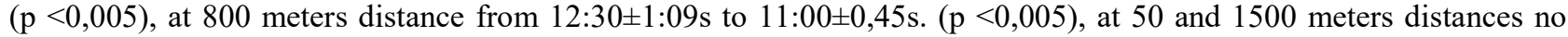
significant changes were revealed. Among the athletes of $\left(n_{3}-7\right)$ group had the most stable sports indices increase at 800 meters distance from $12: 33,7 \pm 0,55,4 \mathrm{~s}$. to $10: 45,4 \pm 0,47,2 \mathrm{~s}$. ( $\mathrm{p}<0,005$ ), at 1500 meters from $24: 10,3 \pm 1: 31,3 \mathrm{~s}$. to 20:05,2 $\pm 1: 02,5 \mathrm{~s} .(\mathrm{p}<0,005)$, at short and average distances this group of athletes didn't have valid changes.

As a result of the tests for coordination - shuttle run $4 \times 9$ meters in $\left(n_{1}-9\right)$ and $\left(n_{2}-16\right)$ groups there were no significant changes at the end of the academic year from $8,93 \pm 0,47 \mathrm{~s}$. to $8,70 \pm 0,51 \mathrm{~s}$., at the same time the swimmers from $\left(n_{3}-7\right)$ group had considerable shuttle run results increase from $9,29 \pm 0,48$ to $8,74 \pm 0,32 \mathrm{~s}$. $(\mathrm{p}<0,005)$.

According to the results of analytical handling we revealed correlational connection of body mass index and control results at free style distances. On the basis of body mass index (BMI) it was defined that in the first group it was $\mathrm{p}_{1}=16,2 \pm 0,35 \mathrm{cu}$ and had statistically significant correlation with the results at short distances $\mathrm{r}=0,477$.; in the second group the athletes with BMI $\mathrm{p}_{2}=17,9 \pm 1,4 \mathrm{cu}$, mostly correlated with the indices at the average distances $\mathrm{r}=0,539$ and had less clear interaction at short distances $\mathrm{r}=0,315$; and in the $3^{\text {rd }}$ group, where body mass index was $\mathrm{p}_{3}=18,3 \pm 1,4$ $\mathrm{cu}$, correlation was with the results at long distances $\mathrm{r}=0,575$.

If we consider the level of adaptation to physical loads according to the research results at bicycle ergometer we revealed that athletes of the accelerated type achieved submaximal HR indices in case of the load $100 \mathrm{~W}$ during the $10^{\text {th }}$ minute of step increasing load for $25 \mathrm{~W}$ each 2 minutes, among $\left(\mathrm{n}_{2}-16\right)$ and $\left(\mathrm{n}_{3}-7\right)$ group athletes submaximal HR indices were defined in terms of the load $100 \mathrm{~W}$ during the $13^{\text {th }}$ minute of testing. Physical working capacity was estimated by means of submaximal HR calculation - $85 \%$ from the calculated maximal HR. The way of maximal HR calculation: subtract the age from 220 .

\section{Conclusions}

The held research proves that pubertal period in 13-14 year-old boys has typical peculiarities. They should be taken into consideration during educational-training lessons planning and competitive distances distribution. Functional reserves of cardio-vascular system determination would help to decrease the risk of disadaptive reactions development to physical load of competitive character, estimate effectively potentialities of athletes depending on the intensity of hormonal-gender changes. Experimentally revealed effectiveness correlation of the distances overcoming with BMI corresponds with the data and resume of the leading specialists. Nevertheless, quick morpho-functional changes of an organism during the studied period demand constant medical and pedagogical control.

\section{References}

1. N.V. Ermolina, O.V. Morozova, A.V. Dorontsev, Yu.A. Lyamina, European Social Science Journal, 2-1, $152-161$ (2018)

2. D.F. Mosunov, Scientific notes of P.F. Lesgaft University, 4(146), 138-144 (2017)

3. I.V. Pyzhova, S.N. Petrova, I.V. Sidorova, Health - the base for personal potential: problems and ways of their solution, 13(2), 676-681 (2018)

4. S.V. Pogodina, G.D. Aleksanyants, Physical culture, sport - science and practice, 3, 68-73 (2018)

5. S.V. Pogodina, A.I. Pogrebnoy, V.S. Yuferev, M.M. Shestakov, Functional parameters of adaptive systems among different age swimmers (KSU PCST, Krasnodar, 2018)

6. A.A. Svetlichkina, A.V. Dorontsev, Scientific notes of P.F. Lesgaft University, 1(143), 181-184 (2017)

7. I.S. Syrvacheva, K.G. Rubis, Materials of the International scientific-practical conference "Urgent problems of physical culture development: theory and practice”, Saint-Petersburg, April, 2015 (Saint-Petersburg, 229-235, 2019)

8. V.R. Solomatin, Physical culture: upbringing, education, training, 3, 41-46 (2010)

9. M.A. Chichkova, A.A. Svetlichkina, A.V. Dorontsev, Scientific notes of P.F. Lesgaft University, 7(149), $203-207$ (2017)

10. G. Aleksanyants, S. Pogodina, V. Yuferev, I. Epishkin, Bulletin of the Georgian national academy of sciences, 12(4), 13-19 (2018)

11. C.A. Kalva-Filho, A. Toubekis, A.M. Zagatto, Pediatric exercise science, 30(3), 383-392 (2017)

12. D.J. Plews, B. Scott, M. Altinib, International journal of sports physiology and performance, 12, 1324-1328 (2017)

13. V.Yu. Karpov, S.Yu. Zavalishina, A.V. Dorontsev, K.K. Skorosov, D.A. Ivanov, Indian Journal of Public Health Research and Development, 10(10), 2723-2728 (2019) 
14. S.V. Pogodina, G.D. Aleksanyants, Physical Culture, Sport - Science and Practice, 3, 68-73 (2018)

15. M.A. Chichkova, A.A. Svetlichkina, A.V. Dorontsev, The Russian Journal of Physical Education and Sport, 13(1), 198-205 $(2018$

16. D.J. Plews, B. Scott, M. Altinib, International journal of sports physiology and performance, 12, 1324-1328 (2017)

17. P. Wahl, C. Manunzio, F. Vogt, J Strength Cond Res, 31(12), 3489-3496 (2017) 